\title{
REVIEW
}

\section{Clinical review: Special populations - critical illness and pregnancy}

Patrick J Neligan ${ }^{* 1,2}$ and John G Laffey ${ }^{1,2}$

\begin{abstract}
Critical illness is an uncommon but potentially devastating complication of pregnancy. The majority of pregnancyrelated critical care admissions occur postpartum. Antenatally, the pregnant patient is more likely to be admitted with diseases non-specific to pregnancy, such as pneumonia. Pregnancy-specific diseases resulting in ICU admission include obstetric hemorrhage, pre-eclampsia/eclampsia, HELLP (hemolysis, elevated liver enzymes, and low platelet count) syndrome, amniotic fluid embolus syndrome, acute fatty liver of pregnancy, and peripartum cardiomyopathy. Alternatively, critical illness may result from pregnancy-induced worsening of pre-existing diseases (for example, valvular heart disease, myasthenia gravis, and kidney disease). Pregnancy can also predispose women to diseases seen in the non-pregnant population, such as acute respiratory distress syndrome (for example, pneumonia and aspiration), sepsis (for example, chorioamnionitis and pyelonephritis) or pulmonary embolism. The pregnant patient may also develop conditions co-incidental to pregnancy such as trauma or appendicitis. Hemorrhage, particularly postpartum, and hypertensive disorders of pregnancy remain the most frequent indications for ICU admission. This review focuses on pregnancy-specific causes of critical illness. Management of the critically ill mother poses special challenges. The physiologic changes in pregnancy and the presence of a second, dependent, patient may necessitate adjustments to therapeutic and supportive strategies. The fetus is generally robust despite maternal illness, and therapeutically what is good for the mother is generally good for the fetus. For pregnancy-induced critical illnesses, delivery of the fetus helps resolve the disease process. Prognosis following pregnancy-related critical illness is generally better than for age-matched non-pregnant critically ill patients.
\end{abstract}

\section{Introduction}

Pregnancy is a normal physiologic process that is defined by the presence of the utero-placental complex. Physiologic changes associated with pregnancy may result in strain on organ systems with limited reserve and result in deterioration of pre-existing medical conditions (Table 1). In addition, a number of systemic disorders result from pathologies of the maternal-fetal interface. The pregnant patient may be admitted to intensive care because of diseases that occur only in pregnancy (Table 2), diseases that are worsened by pregnancy resulting in critical illness, diseases for which the pregnant patient is at elevated risk, and diseases co-incidental to pregnancy. This review will concentrate on pregnancy-induced diseases.

\footnotetext{
*Correspondence: patrick.neligan@hse.ie

'Department of Anaesthesia and Intensive Care Medicine, Galway University

Hospitals, Newcastle Road, Galway, Co. Galway, Ireland

Full list of author information is available at the end of the article
}

\section{Obstetrical hemorrhage}

Major obstetric hemorrhage is the leading cause of maternal mortality worldwide and is the most frequent indication for ICU admission [1-3]. It may occur antepartum or postpartum.

\section{Antepartum hemorrhage}

Antepartum hemorrhage occurs in 1 in 20 pregnant women; in the majority of cases, there is no risk to the mother or fetus. Causes include abruptio placentae, placenta previa, placenta accreta/increta/percreta, and uterine rupture. Abruptio placentae (placental abruption) involves separation of the placenta from the decidua basalis prior to delivery. The patient may present with pain, vaginal bleeding, uterine tenderness, and increased uterine activity. Fetal heart rate abnormalities may be encountered. Depending on the location of bleeding, considerable blood loss may occur prior to diagnosis. Significant hemorrhage is associated with coagulopathy. Placenta previa involves implantation of the placenta in the lower part of the uterus and often is associated with a previous Cesarean section (CS) scar. Placenta accreta is 


\begin{tabular}{|c|c|}
\hline System & Changes \\
\hline Respiratory system & $\begin{array}{l}\text { Increased alveolar ventilation }(70 \%) \\
\text { Relative hypocarbia }\left(\mathrm{PaCO}_{2} \text { of } 25-32 \mathrm{~mm} \mathrm{Hg}\right) \\
\text { Reduced functional residual capacity }(20 \%) \\
\text { Increased } \mathrm{O}_{2} \text { consumption } \\
\text { Reduced venous oxygen saturation }\left(\mathrm{SvO}_{2}\right)\end{array}$ \\
\hline Cardiovascular system & $\begin{array}{l}\text { Increased cardiac output (40\%) } \\
\quad \text { Increased stroke volume } 25 \% \text {, increased heart rate 25\% } \\
\text { Reduced total peripheral resistance } \\
\text { Normal CVP in superior vena cava distribution } \\
\text { Elevated CVP in inferior vena cava distribution } \\
\quad \text { Aorto-caval compression } \\
\text { Increased circulating volume } \\
\text { Increased plasma volume (40\%-50\%) } \\
\text { Increased red cell mass (20\%) } \\
\quad \text { Physiologic anemia }\end{array}$ \\
\hline Gastrointestinal and metabolic & $\begin{array}{l}\text { Reduced lower esophageal sphincter tone } \\
\text { Elevated risk of gastro-pulmonary aspiration } \\
\text { Increased metabolism } \\
\text { Carbohydrate +++ } \\
\text { Protein ++ } \\
\text { Fat + } \\
\text { Hyperglycemia (due to insulin resistance) }\end{array}$ \\
\hline Kidney & $\begin{array}{l}\text { Increase renal blood and plasma flow (50\%-60\%) } \\
\text { Increased glomerular filtration (50\%-60\%) } \\
\text { Reduced serum urea and creatinine } \\
\text { Glycosuria } \\
\text { Mild proteinuria }\end{array}$ \\
\hline Hematopoietic system & $\begin{array}{l}\text { Reduced hemoglobin concentration (functional anemia despite elevated red cell mass) } \\
\text { Slightly elevated leucocyte count } \\
\text { Slightly reduced platelet count } \\
\text { Increased clotting tendency }\end{array}$ \\
\hline
\end{tabular}

$\mathrm{CVP}$, central venous pressure; $\mathrm{PaCO}_{2}$, arterial partial pressure of carbon dioxide.

an abnormally adherent placenta that implants in the uterine wall, usually in scar tissue following previous CS. With increasing severity there is placenta increta invasion of the myometrium and placenta percreta - in which the placenta invades the extra-uterine pelvic tissues. Uterine rupture during labor is another potential complication and is infrequently associated with previous CS.

\section{Postpartum hemorrhage}

Postpartum hemorrhage (PPH) involves blood loss of greater than $500 \mathrm{~mL}$ within 24 hours regardless of the mode of birth. However, there is no universally accepted definition, and consideration should be given to physiologic response in addition to absolute blood loss. PPH is the most frequent indication for ICU admission. In $60 \%$ to $70 \%$ of cases, the cause of $\mathrm{PPH}$ is failure of uterine contraction following delivery. This uterine atony results in continuous bleeding that is often painless. Placental retention is the second most common cause of PPH $(20 \%$ to $30 \%$ of cases). Genital trauma results in approximately $10 \%$ of cases of $\mathrm{PPH}$ and usually is associated with laceration of the vagina/cervix following instrumental delivery. Coagulation disorders may also result in PPH.
These may be congenital - hemophilia or von Willebrand disease - or acquired: sepsis, amniotic fluid embolus (AFE) syndrome, acute fatty liver of pregnancy, preeclampsia, or HELLP (hemolysis, elevated liver enzymes, and low platelet count) syndrome.

\section{Management}

An aggressive coordinated multidisciplinary approach between obstetricians, midwives, anesthesiologists, the laboratory, and blood bank is required (Figure 1). Initial management depends on the cause of the hemorrhage and whether delivery of the fetus has occurred. Where the hemorrhage occurs postpartum, uterine atony with or without retained products should be suspected. Oxytocin should be administered, the bladder should be emptied, and the uterus massaged. Intravenous access is obtained, blood is sent for crossmatching, and if crossmatched blood is unavailable, $\mathrm{O}$ negative blood is obtained. The obstetrician should examine the genital tract for evidence of trauma. If bleeding persists, prostaglandin therapy - either intravenous prostaglandin $\mathrm{E}_{2}$ or 15 -methyl prostaglandin $\mathrm{F}_{2 \alpha}-$ is administered. Consideration should be given to uterine tamponade, by packing or balloon compression. Continued bleeding should result 
Table 2. Diagnoses that may result in intensive care admission during pregnancy and in the puerperium

Pregnancy-related

Obstetrical hemorrhage

Antepartum

Placental abruption

Placenta previa

Placenta accreta

Postpartum

Retained placenta

Failure of uterine contraction

Trauma

Coagulopathy

Pre-eclampsia

Eclampsia

HELLP syndrome

Acute fatty liver of pregnancy

Peripartum cardiomyopathy

Sepsis

Chorioamnionitis

Endometritis

Amniotic fluid embolism syndrome

Pre-existing diseases that may worsen during pregnancy

Neurologic

Epilepsy

Myasthenia gravis

Cardiovascular

Valvular disease

Primary pulmonary hypertension

Congenital heart disease

Endocrine

Renal

Diabetes mellitus

Acceleration of chronic kidney disease

Diseases for which the pregnant patient has increased risk Sepsis

Urinary tract infection (particularly pyelonephritis)

Listeriosis

Pneumonia

Endocrine

Diabetic ketoacidosis

Sheehan syndrome (secondary to peripartum hemorrhage)

Pulmonary

Pulmonary embolism

Aspiration pneumonitis

Hematologic

Deep venous thrombosis

Disseminated intracascular coagulopathy

Renal

Acute kidney injury (variety of mechanisms)

Acute on chronic renal failure

Diseases that may be co-incidental to pregnancy

Trauma

Penetrating

Blunt trauma

Surgical illness

Ruptured intracranial aneurysm

Appendicitis

Cholecystitis

HELLP, hemolysis, elevated liver enzymes, and low platelet count. in surgical intervention: arterial ligation, B-Lynch suture, Cesarean hysterectomy, or uterine artery embolization [4]. It is important that these interventions occur without delay; with each advancing minute, continued blood loss leads to loss of clotting factors, coagulopathy, and the potential for exsanguination. It is imperative that the patient be kept warm and that blood products and fluids be warmed through a rapid transfusion device. An arterial line should be placed to measure blood pressure and pulse pressure variability and to take blood samples to track hematocrit and acid-base status. Ideally, qualitative tests of clot quality (for example, thromboelastography) would be combined with coagulation studies to guide blood component resuscitation. In the event of uncontrolled non-surgical bleeding, consideration should be given to the administration of recombinant factor VIIa ( $90 \mu \mathrm{g} / \mathrm{kg}$, repeated at 20 minutes if there is no response) [5-7].

\section{Pre-eclampsia and eclampsia}

Pre-eclampsia toxemia (PET) is a multisystem disease characterized by impaired organ perfusion resulting from vasospasm and activation of the coagulation system. PET is defined as hypertension and proteinuria appearing after the 20th week of gestation and resolving within 6 to 12 weeks of delivery. It occurs in $2 \%$ to $3 \%$ of all pregnancies and is more common in primigravida or the first pregnancy with a particular partner. Other risk factors include a positive family history, pre-existing hypertension, diabetes mellitus, multiple pregnancy, increasing maternal age, and obesity.

The pathogenesis of PET appears to result from abnormal placenta formation (termed 'placentation'). Failure of the second phase of trophoblast invasion results in the lack of destruction of the muscularis layer of the spiral arterioles, impairing vasodilation in response to increases in blood flow, resulting in placental ischemia. The ischemic placenta releases vasoactive substances, leading to systemic endothelial injury and systemic organ dysfunction. As pregnancy progresses, placenta ischemia worsens, and the mother becomes hypovolemic and hypertensive and may develop renal dysfunction. There is disordered prostaglandin metabolism, with an imbalance between vasoconstrictive thromboxane and vasodilatory prostacyclin, resulting in platelet dysfunction, endothelial damage, and further vasoconstriction.

PET is classified as mild, moderate, or severe. Severe pre-eclampsia is defined as one of the following: (a) severe hypertension (systolic blood pressure of greater than $160 \mathrm{~mm} \mathrm{Hg}$ or diastolic blood pressure of greater than $110 \mathrm{~mm} \mathrm{Hg}$ ), (b) proteinuria of greater than $5 \mathrm{~g}$ per 24 hours, (c) oliguria of less than $400 \mathrm{~mL}$ per 24 hours, (d) cerebral irritability, (e) epigastric or right upper quadrant pain (liver capsule distension), or (f) pulmonary 


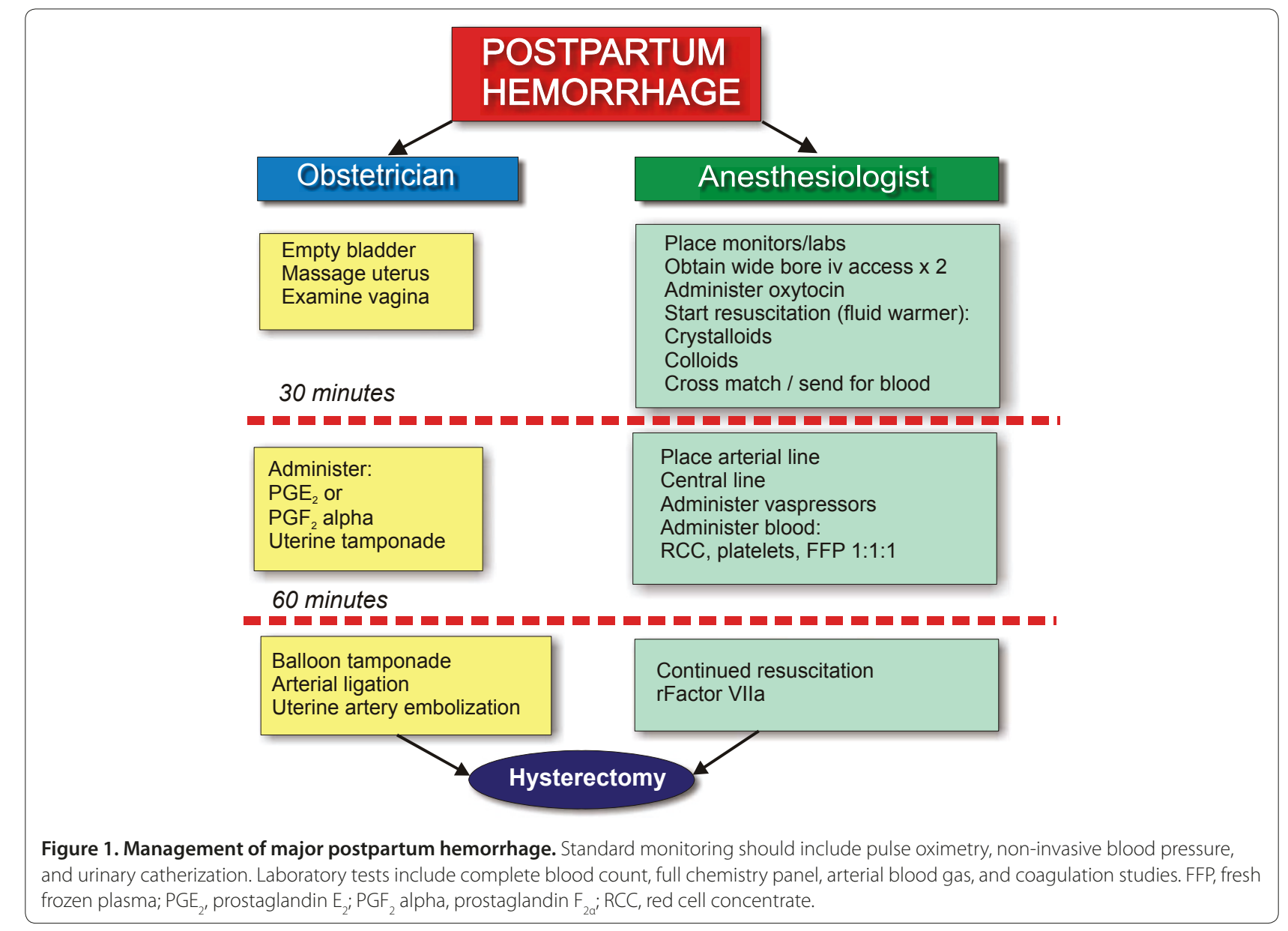

edema. PET is associated with significant morbidity and mortality, for the mother and fetus. PET usually resolves following delivery of the fetus but may manifest postpartum. A variety of antihypertensive agents, including hydralazine, labetalol, sodium nitroprusside, alpha blockers, calcium channel blockers, and methyl dopa, have been advocated in PET. Hydralazine and labetalol are the most widely used of these in the critical care setting. Hydralazine is administered by slow intravenous injection: first with $5 \mathrm{mg}$ intravenously and repeated at 20-minute intervals (5 to $10 \mathrm{mg}$ depending on the response). Once blood pressure control is achieved, hydralazine can be repeated as needed (usually about every 3 hours). Labetolol is started at $20 \mathrm{mg}$ intravenously as a bolus, and the dose is doubled every 10 minutes until blood pressure is under control. The maximum dose is $220 \mathrm{mg}$. Magnesium is usually co-administered to provide vasodilatation and prevent seizures. Care should be taken with fluid resuscitation because of the risk of pulmonary edema.

Eclampsia is an extreme complication of PET and is defined by the occurrence of seizures in the absence of other neurologic disorders. Up to $40 \%$ of seizures occur following delivery. Convulsions are believed to result from severe intracranial vasospasm, local ischemia, intracranial hypertension, and endothelial dysfunction associated with vasogenic and cytotoxic edema. Seizures tend to be self-limiting, and status epilepticus is unusual. Magnesium sulphate $\left(\mathrm{MgSO}_{4}\right)$ is superior to phenytoin and benzodiazepines in the prevention of recurrent eclamptic seizures [8]. Magnesium toxicity is rare in the absence of renal failure. Respiratory arrest caused by magnesium toxicity can be reversed with calcium. While traditionally the mortality from eclampsia has been high, death is now uncommon, and much of the mortality is attributable to hepatic complications, including hepatic failure, hemorrhage, or infarction [9].

\section{HELLP syndrome}

HELLP syndrome is a constellation of findings that include hemolysis with a microangiopathic hemolytic anemia (hemolysis), elevated liver function tests, and thrombocytopenia (low platelets). HELLP syndrome complicates up to 3 per 1,000 pregnancies, may present as a severe manifestation of PET, and occurs in up to $20 \%$ of patients. The majority of patients are diagnosed before 
37 weeks of gestation. There is a clear overlap between PET and HELLP syndrome, and it is unclear whether the latter is a primary or secondary disease process. The syndrome is believed to be due to generalized endothelial and microvascular injury from activation of the complement and coagulation cascades, increased vascular tone, and platelet aggregation. This results in areas of hemorrhage and necrosis within the liver and may evolve to large hematomas, capsular tears, and intraperitoneal bleeding. Laboratory criteria for this syndrome include microangiopathic hemolysis with schistocytes present on the peripheral blood smear, platelet count of fewer than $50,000 / \mathrm{mm}^{3}$, serum total bilirubin of more than $20 \mu \mathrm{mol} / \mathrm{L}$ $(1.2 \mathrm{mg} / 100 \mathrm{~mL})$, serum lactate dehydrogenase of more than $600 \mathrm{U} / \mathrm{L}$, and serum aspartate transaminase of more than $70 \mathrm{U} / \mathrm{L}$.

The differential diagnosis includes thrombotic thrombocytopenic purpura and hemolytic uremic syndrome, cold agglutinins, and acute fatty liver of pregnancy. In regard to distinguishing these conditions, it is likely that the patient with HELLP syndrome will have a more severe liver dysfunction, potentially complicated by hepatic infarction or subcapsular hematoma. HELLP syndrome is a medical emergency and the mother's blood pressure and coagulation status must be stabilized rapidly. Corticosteroids should be administered to the mother to advance the lung maturity of the fetus if the gestation age is below 34 weeks. In the majority of cases, liver, renal, and hematopoietic function normalizes after 5 days. In about $30 \%$ of cases, HELLP syndrome will develop in the postpartum period. Dexamethasone does not benefit the mother with HELLP syndrome [10]. Delivery of the fetus can significantly abrogate HELLP syndrome. The timing of delivery depends on fetal maturity and the severity of illness of the mother.

The major life-threatening complications of HELLP syndrome are hepatic hemorrhage, subcapsular hematoma, liver rupture, and multi-organ failure. Liver hemorrhage is managed conservatively where possible with aggressive blood product resuscitation to reverse the coagulopathy and ensure adequate oxygen-carrying capacity. The development of a subcapsular hematoma may lead to hepatic rupture, which is potentially life-threatening for the mother and fetus, and 50\% maternal and up to $60 \%$ fetal mortality rates have been reported. Management of threatened or actual hepatic rupture involves drainage of the hematoma, packing, oversewing of lacerations, or partial hepatectomy. Consideration should be given to hepatic arterial embolization, either in the high-risk patient or in the period following operative stabilization.

\section{Acute fatty liver of pregnancy}

Acute fatty liver disease of pregnancy (AFLP) occurs in about 1 per 10,000 pregnancies characterized by hepatic microvesicular steatosis and manifests in the third trimester. Without early diagnosis and treatment (fetal delivery), the patient may develop acute liver failure and hepatic encephalopathy. It is more common in primiparas, in twin pregnancies, and in patients who have preeclampsia. AFLP is a mitochondrial disorder [11] related to inherited mutations that cause a deficiency of the long-chain 3-hydroxyacyl coenzyme A dehydrogenase (LCHAD), a fatty acid beta-oxidation enzyme. When a heterozygous mother has a fetus that is homozygous for these mutations, the fetus is unable to metabolize longchain fatty acids; these acids accumulate in the fetus and spill over into the maternal circulation [12]. This mutant gene and deficient coenzyme product leads to accumulation of long-chain fatty acid metabolites that are hepatotoxic [13].

The patient usually presents with vague symptoms, vomiting, or abdominal pain and may develop preeclampsia. Often there are no specific clinical signs, except tenderness in the right upper quadrant. Serum aminotransferase and bilirubin levels are significantly elevated, and in later stages there is a coagulopathy evidenced by low fibrinogen and a prolonged prothrombin time. Compared with HELLP, thrombocytopenia and hypertension are unusual. Urate levels may be extremely high and there may be significant hypoglycemia. Conclusive diagnosis requires liver biopsy, although owing to coagulopathy this is rarely possible or practical. The differential diagnosis includes HELLP syndrome, pre-eclampsia, and acute hepatitis due to alcohol or a virus [14]. The treatment of choice is urgent delivery of the fetus. This stops the overload of the mother's fatty acid oxidation system from fetal production and leakage into the maternal circulation [13].

\section{Amniotic fluid embolus syndrome}

AFE syndrome is a devastating complication that usually occurs within 24 hours of delivery. It manifests with acute severe hypoxic respiratory failure, associated with shock, disseminated intravascular coagulopathy (DIC), confusion, and seizures (Table 3 and Figure 2). The incidence of AFE syndrome is unclear, and reports in the literature vary between 1:8,000 and 1:80,000 deliveries [15]. The disease is likely under-reported because of the absence of clear diagnostic criteria. Similarly, the mortality rate with AFE syndrome has been reported to be as high as $85 \%$, and the majority of survivors suffer chronic neurologic deficit [16].

The pathophysiology of AFE syndrome is unclear. Previously, this syndrome was believed to result from embolization of amniotic fluid into the pulmonary circulation. However, an anaphylactoid or hypersensitivity reaction to the contents of this fluid is more likely [17]. The presence of amniotic fluid in the pulmonary 
Table 3. Clinical features of amniotic fluid embolus syndrome

Acute hypoxic respiratory failure (acute lung injury)

Coagulopathy/Bleeding

Hypotension/Shock

Right heart failure

Confusion/Seizures

circulation is neither sensitive nor specific, as this has been identified in mothers who do not develop AFE syndrome. Patients may present with seizure-type activity or acute respiratory distress. Acute lung injury results in profound hypoxemia, intense hypoxic pulmonary vasoconstriction, and acute right heart failure (Table 4), resulting in hemodynamic collapse. Bowing of the right ventricle into the left results in acute diastolic and then acute systolic failure of the left ventricle. There is simultaneous DIC that may manifest with bleeding from the placental bed. Nausea, vomiting, headache, confusion, and seizures commonly follow. Death from AFE syndrome results from multi-organ failure, exsanguinations, or cardiac arrest. Neurologic injury is common in survivors.

There is no diagnostic test for AFE syndrome. In a high-risk peripartum patient (Table 4) or one who has recently undergone termination of pregnancy (with hypertonic saline), the combination of coagulopathy, ARDS, and shock should be considered AFE syndrome until otherwise proven. The differential diagnosis includes sepsis, particularly due to chorioamniitis, thromboembolic pulmonary embolism, and aspiration pneumonitis. The fetus should be delivered emergently to avoid fetal demise. CS may be complicated by excess bleeding, requiring ligation of the uterine arteries and perhaps hysterectomy. There is no specific treatment, although both aprotinin and activated protein $\mathrm{C}$, compounds that modulate inflammation and coagulation, may have some

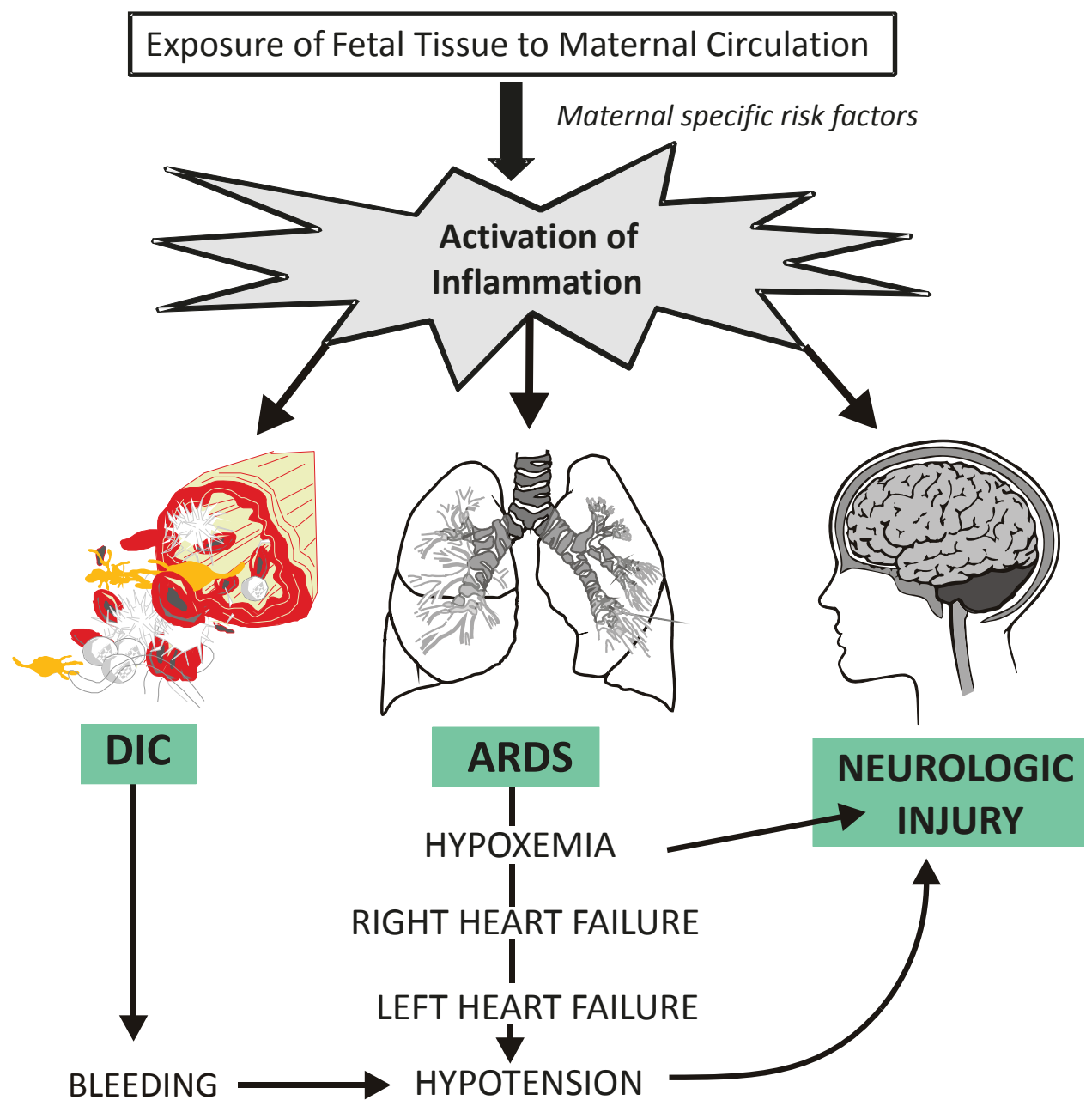

Figure 2. Amniotic fluid embolism. ARDS, acute respiratory distress syndrome; DIC, disseminated intravascular coagulopathy. 


\begin{tabular}{l} 
Table 4. Risk factors for the development of amniotic fluid \\
embolus syndrome \\
\hline Timing: during labor and delivery or immediately postpartum \\
Cesarean section \\
Amniocentesis \\
Trauma \\
Multiparity \\
Termination of pregnancy by using hypertonic saline \\
Advanced maternal age \\
Prolonged gestation \\
Male fetus
\end{tabular}

utility [18]. Critical care management should be directed at maintaining oxygen delivery and supporting the heart and circulation with inotropes and vasopressors. Early echocardiography is extremely useful to determine the nature of the cardiac injury (right versus left ventricular failure). Right ventricular failure can be worsened by high levels of positive end-expiratory pressure and vasopressors and can be managed with milrinone, enoxamone, dobutamine, inhaled nitric oxide, and nebulized prostacyclin. Very large quantities of blood products may be required to control the coagulopathy, and this can result in significant fluid overload. Early consideration should be given to continuous renal replacement therapy. Although extracorporeal membrane oxygenation may appear to be an ideal approach to cardiopulmonary failure, excess bleeding may limit its application [19].

\section{Peripartum cardiomyopathy}

Peripartum cardiomyopathy is defined as a dilated cardiomyopathy of unknown cause associated with pregnancy. It occurs in the last gestational month or in first 5 months postpartum and is associated with no other cardiac disease [20]. Although this condition is relatively rare, its exact incidence is unclear (the reported incidence varies between 1 in 1,500 and 1 in 15,000 pregnancies), and the condition is more common among Africans and Haitians. It is associated with older maternal age, obesity, multiparity, multiple pregnancies, and pregnancy-induced hypertension. The patient typically presents with symptoms of congestive heart failure: dyspnea, orthopnea, shortness of breath on exertion, upper abdominal pain, and so on. Echocardiography demonstrates systolic dysfunction.

Proposed pathogenic hypotheses for peripartum cardiomyopathy include viral myocarditis, auto-immunemediated injury, and prolonged tocolysis. Current evidence strongly suggests that the disease is triggered by a by-product of prolactin metabolism, resulting in unbalanced peri-/postpartum oxidative stress [21]. The hormone is proteolytically cleaved, and an antiangio- genic, proapoptotic, and proinflammatory 16-kDa byproduct appears to attack the myocardium [22].

Medical therapy is effective in the majority of patients; treatment is commenced with a loop diuretic, and if the patient is postpartum, an angiotensin-converting enzyme inhibitor or antiotensin receptor blocker is added. In severe cases, placement of an intra-aortic balloon counterpulsation device or extracorporeal membrane oxygenation [23] may be necessary. There is a very high incidence of thromboembolic disease associated with peripartum cardiomyopathy, and anticoagulation is essential. Early experience with prolactin inhibitors, such as bromocriptine, appears to be positive, and this may become the mainstay of treatment in the future [24].

Mortality appears to be high; in the US, mortality occurs in $25 \%$ to $50 \%$ of cases, usually within 3 months of diagnosis [25]. Availability of quality health care for the vulnerable population may be a component of this. Approximately $50 \%$ of women recover their ventricular function within 6 months of delivery. In some cases, cardiac transplantation is necessary. Despite recovery, cardiomyopathy may recur in subsequent pregnancies.

\section{Sepsis}

Pregnancy predisposes women to four specific infectious complications: pyelonephritis, chorioamnionitis (including septic abortion), endometritis (often following Cesarean delivery), and pneumonia. Pyelonephritis results from colonization of the kidney with Gram-negative bacteria secondary to loss of ureteral sphincter tone associated with progesterone. Pneumonia results, at least in part, from aspiration of gastric contents as a consequence of loss of lower esophageal sphincter tone and diaphragmatic elevation. Patients are also at elevated risk for viral and fungal pneumonia due to pregnancy-induced immunosuppression. Chorioamnionitis results from alterations in the $\mathrm{pH}$ and increased glycogen content of the vagina, resulting in loss of the barrier for bacterial entry. It may complicate chorionic villus sampling, amniocentesis, or attempted instrumental (septic) abortion.

Bacteremia in pregnancy is relatively common (reportedly occurring in $8 \%$ to $9 \%$ of pregnancies), whereas progression to severe sepsis and septic shock is relatively rare [26-29]; the rate of sepsis ranges from 1 in 7,654 to 1 in 8,338 deliveries reported [30]. Kankuri and colleagues [31] reported that only 1 of 43,483 mothers developed septic shock during the peripartum period. Despite these low progression rates, the mortality from sepsis in pregnancy remains significant. Infections may be Gramnegative, Gram-positive, or rarely anerobic in etiology. The most commonly isolated organisms are Escherichia coli, enterococci, and beta hemolytic streptococci.

The majority of infections occur postpartum; 'puerpural sepsis' or 'puerperal fever' is an umbrella term for a 
variety of infections that occur in the puerperium. The leading risk factor for puerpural sepsis is Cesarean delivery. Other significant risk factors include retained products of conception, episiotomy, and prolonged rupture of the amniotic membranes. Infection may involve endometritis, parametritis (spread through the uterine wall), peritonitis, or thrombophlebitis of the pelvic veins. Endometritis is most commonly associated with group A streptococcal (GAS) infection, although Staphylococus, coliform, and anerobes may also be present. Hand washing and disinfectants dramatically decreased the incidence of puerperal fever. Pre-emptive antibiotics are administered if prolonged rupture of the membranes has occurred, to treat amnionitis, or if the woman has a fever and a foul-smelling vaginal discharge.

The most recent maternal mortality report from the UK revealed that sepsis was the leading cause of maternal death between 2006 and 2008 [32]. Sepsis resulted in 29 maternal deaths. Fifty percent of deaths occurred following Cesarean delivery, whereas 7 occurred after vaginal delivery. One third of these deaths occurred before 24 weeks of gestation. There was a marked seasonal pattern; most deaths occurred between December and April. Associated factors included minority ethnic origin and the presence of sickle cell disease or trait. Interestingly, obesity was not a risk factor. Infant mortality in affected pregnancies was $45 \%$. GAS was the principal pathogen, causing nearly $50 \%$ of deaths. All mothers who died from GAS either worked with or had children, and most of the mothers had a direct or family history of sore throat or respiratory infection. In the developing world, the mortality rate from puerperal sepsis remains extremely high (greater than $70 \%$ ) and tetanus is a common cause of infection.

The clinical manifestations of sepsis include the hallmarks of systemic inflammation that may be followed by coagulopathy, vasoplegia, and evolving multiple organ failure. The patient typically has a temperature of $38^{\circ} \mathrm{C}$ during the period from the end of the first to the end of the 10th day after childbirth or abortion. Purpura fulminans may be associated with GAS infection. Physicians must have a high index of suspicion for sepsis in any peripartum patient who presents with fever and evidence of organ dysfunction: confusion, oliguria, tachycardia, and so on. Treatment includes fluid resuscitation, empiric antibiotic therapy, and source control. Early aggressive volume resuscitation is essential and is followed with vasopressor therapy if necessary. There is no evidence to suggest that norepinephrine has an adverse effect on fetal well-being. There are no data on the use of vasopressin during pregnancy. Considering current data and the specific exclusion of pregnant (but not postpartum) patients from the PROWESS (Recombinant Human Activated Protein C Worldwide Evaluation in Severe Sepsis) trial and other trials, we cannot recommend the use of activated protein $\mathrm{C}$ during pregnancy. However, this agent may be of value in the postpartum period and clinicians should weigh up its value as a potent anti-inflammatory/anticoagulant agent versus the risk of bleeding complications.

Goal-directed resuscitation of the pregnant patient is recommended. One must be aware of physiologic changes and adjust the goals of the resuscitation accordingly. Although central venous pressure remains essentially unchanged during pregnancy, venous oxygen saturation $\left(\mathrm{SvO}_{2}\right)$ progressively decreases in the later stages. Hence, achieving goals of $70 \%$ to $75 \%$ may not be possible - or necessary - in this setting. Antimicrobials are administered on the basis of the 'best guess' source of infection. In general, tetracycline and quinolone antibiotics should be avoided in early pregnancy. Penicillins, macrolides, and cephalosporins appear relatively safe within normal dosage range.

\section{Cardiac arrest and brain death}

When cardiac arrest occurs in late pregnancy, cardiopulmonary resuscitation (CPR) is difficult [33]. The airway should be secured without delay, and the patient should be positioned to ensure left lateral displacement of the uterus. Unfortunately, this results in less effective CPR [33]. Drugs and defibrillation regimens should follow standard Advanced Cardiovascular Life Support guidelines. A decision regarding Cesarean delivery should be made immediately. Current data suggest that this should be performed within 5 minutes, to ensure viability of the mother [34]. Extraction of the fetus results in an increase in maternal blood volume and release of aortocaval compression [35]. This should be undertaken from the second trimester onwards. If the decision to perform Cesarean delivery is made, there should be no delay and CPR should be continued throughout surgery.

Maternal brain death, due, for example, to an intracranial bleed, raises difficult ethical and legal issues $[36,37]$. Depending on the gestational age, it may be possible, if the fetus is near viability, to maintain maternal organ support to allow the fetus to reach viability [38]. Although it may appear expensive to sustain the vegetative state of the brain-dead mother, this cost may be significantly less than the long-term cost of care of a severely premature neonate, which would require neonatal ICU and a variety of health-care interventions.

\section{Conclusions}

Critical illness is an uncommon but potentially devastating complication of pregnancy. The first priority for the intensivist is to stabilize the mother, with the understanding that what is good for the mother is good for the fetus. The intensivist must be aware of the 
physiologic changes associated with pregnancy and their timing. Critical care interventions are similar to those for the non-pregnant patient; however, adjustment of physiologic targets for metabolic, pulmonary, and hemodynamic control may be necessary. The majority of acquired pregnancy-related diseases, such as PET, AFLP, and cardiomyopathy, are abrogated by delivery. However, timing and the state of fetal maturation are crucial to decision making.

\section{Abbreviations}

AFE, amniotic fluid embolus; AFLP, acute fatty liver disease of pregnancy; CPR, cardiopulmonary resuscitation; CS, Cesarean section; DIC, disseminated intravascular coagulopathy; GAS, group A streptococcal; HELLP, hemolysis, elevated liver enzymes, and low platelet count; ICU, intensive care unit; PET, pre-eclampsia toxemia; PPH, postpartum hemorrhage.

\section{Competing interests}

The authors declare that they have no competing interests.

\section{Acknowledgments}

This study was funded from Departmental resources.

\section{Author details}

'Department of Anaesthesia and Intensive Care Medicine, Galway University Hospitals, Newcastle Road, Galway, Co. Galway, Ireland. 'Department of Anaesthesia, School of Medicine, Clinical Sciences Institute, National University of Ireland, Galway, Co. Galway, Ireland.

Published: 12 August 2011

\section{References}

1. Sriram S, Robertson MS: Critically ill obstetric patients in Australia: a retrospective audit of 8 years' experience in a tertiary intensive care unit. Crit Care Resusc 2008, 10:124.

2. Hazelgrove JF, Price C, Pappachan VJ, Smith GB: Multicenter study of obstetric admissions to 14 intensive care units in southern England. Crit Care Med 2001, 29:770-775.

3. Tang LC, Kwok AC, Wong AY, Lee YY, Sun KO, So AP: Critical care in obstetrical patients: an eight-year review. Chin Med J (Engl) 1997, 110:936-941.

4. Bodelon C, Bernabe-Ortiz A, Schiff MA, Reed SD: Factors associated with peripartum hysterectomy. Obstet Gynecol 2009, 114:115-123.

5. Wissa I, Ebeid E, El-Shawarby S, Chandakas S, Kamal T, Hill N: The role of recombinant activated Factor VII in major obstetric haemorrhage: the Farnborough experience. J Obstet Gynaecol 2009, 29:21-24.

6. Nohira T, Osakabe Y, Suda S, Takahashi C, Tanaka A, Ikeda K, Ikeda T, Okabe K. Successful management by recombinant activated factor VII in a case of disseminated intravascular coagulopathy caused by obstetric hemorrhage. J Obstet Gynaecol Res 2008, 34:623-630.

7. Welsh A, McLintock C, Gatt S, Somerset D, Popham P, Ogle R: Guidelines for the use of recombinant activated factor VII in massive obstetric haemorrhage. Aust N Z J Obstet Gynaecol 2008, 48:12-16.

8. Duley L: Magnesium sulphate in eclampsia. Eclampsia Trial Collaborative Group Lancet 1998, 352:67-68.

9. Hay JE: Liver disease in pregnancy. Hepatology 2008, 47:1067-1076.

10. Fonseca JE, Mendez F, Catano C, Arias F: Dexamethasone treatment does not improve the outcome of women with HELLP syndrome: a doubleblind, placebo-controlled, randomized clinical trial. Am J Obstet Gynecol 2005, 193:1591-1598.

11. Ibdah JA: Acute fatty liver of pregnancy: an update on pathogenesis and clinical implications. World J Gastroenterol 2006, 12:7397-7404

12. Treem WR, Shoup ME, Hale DE, Bennett MJ, Rinaldo P, Millington DS, Stanley CA, Riely CA, Hyams JS: Acute fatty liver of pregnancy, hemolysis, elevated liver enzymes, and low platelets syndrome, and long chain 3-hydroxyacylcoenzyme A dehydrogenase deficiency. Am J Gastroenterol 1996, 91:2293-2300

13. Cappell MS: Hepatic disorders severely affected by pregnancy: medical and obstetric management. Med Clin North Am 2008, 92:739-viii.
14. Hamid SS, Jafri SM, Khan H, Shah H, Abbas Z, Fields H: Fulminant hepatic failure in pregnant women: acute fatty liver or acute viral hepatitis? J Hepatol 1996, 25:20-27.

15. Clark SL, Hankins GD, Dudley DA, Dildy GA, Porter TF: Amniotic fluid embolism: analysis of the national registry. Am J Obstet Gynecol 1995, 172:1158-1167.

16. Aurangzeb I, George L, Raoof S: Amniotic fluid embolism. Crit Care Clin 2004 20:643-650.

17. Clark SL: New concepts of amniotic fluid embolism: a review. Obstet Gynecol Surv 1990, 45:360-368.

18. Stroup J, Haraway D, Beal JM: Aprotinin in the management of coagulopathy associated with amniotic fluid embolus. Pharmacotherapy 2006, 26:689-693.

19. Hsieh YY, Chang CC, Li PC, Tsai HD, Tsai CH: Successful application of extracorporeal membrane oxygenation and intra-aortic balloon counterpulsation as lifesaving therapy for a patient with amniotic fluid embolism. Am J Obstet Gynecol 2000, 183:496-497.

20. Pearson GD, Veille JC, Rahimtoola S, Hsia J, Oakley CM, Hosenpud JD, Ansari A, Baughman KL: Peripartum cardiomyopathy: National Heart, Lung, and Blood Institute and Office of Rare Diseases (National Institutes of Health) workshop recommendations and review. JAMA 2000, 283:1183-1188.

21. Hilfiker-Kleiner D, Sliwa K, Drexler H: Peripartum cardiomyopathy: recent insights in its pathophysiology. Trends Cardiovasc Med 2008, 18:173-179.

22. Hilfiker-Kleiner D, Kaminski K, Podewski E, Bonda T, Schaefer A, Sliwa K, Forster O, Quint A, Landmesser U, Doerries C, Luchtefeld M, Poli V, Schneider MD, Balligand JL, Desjardins F, Ansari A, Struman I, Nguyen NQ, Zschemisch NH, Klein G, Heusch G, Schulz R, Hilfiker A, Drexler H: A cathepsin D-cleaved $16 \mathrm{kDa}$ form of prolactin mediates postpartum cardiomyopathy. Cell 2007, 128:589-600.

23. Smith IJ, Gillham MJ: Fulminant peripartum cardiomyopathy rescue with extracorporeal membranous oxygenation. Int J Obstet Anesth 2009, 18:186-188.

24. Jahns BG, Stein W, Hilfiker-Kleiner D, Pieske B, Emons G: Peripartum cardiomyopathy--a new treatment option by inhibition of prolactin secretion. Am J Obstet Gynecol 2008, 199:e5-e6.

25. Ro A, Frishman WH: Peripartum cardiomyopathy. Cardiol Rev 2006, 14:35-42.

26. Bryan CS, Reynolds KL, Moore EE: Bacteremia in obstetrics and gynecology. Obstet Gynecol 1984, 64:155-158.

27. Blanco JD, Gibbs RS, Castaneda YS: Bacteremia in obstetrics: clinical course. Obstet Gynecol 1981, 58:621-625.

28. Monif GR, Baer H: Polymicrobial bacteremia in obstetric patients. Obstet Gynecol 1976, 48:167-169.

29. Ledger WJ, Norman M, Gee C, Lewis W: Bacteremia on an obstetricgynecologic service. Am J Obstet Gynecol 1975, 121:205-212.

30. Mabie WC, Barton JR, Sibai B: Septic shock in pregnancy. Obstet Gynecol 1997, 90:553-561.

31. Kankuri E, Kurki T, Carlson P, Hiilesmaa V: Incidence, treatment and outcome of peripartum sepsis. Acta Obstet Gynecol Scand 2003, 82:730-735.

32. Centre for Maternal and Child Enquiries (CMACE): Saving Mothers' Lives: reviewing maternal deaths to make motherhood safer: 2006-08. The Eighth Report on Confidential Enquiries into Maternal Deaths in the United Kingdom. BJOG 2011, 118 (Suppl. 1):1-203.

33. Campbell TA, Sanson TG: Cardiac arrest and pregnancy. J Emerg Trauma Shock 2009, 2:34-42.

34. Zdolsek HJ, Holmgren S, Wedenberg K, Lennmarken C: Circulatory arrest in late pregnancy: caesarean section a vital decision for both mother and child. Acta Anaesthesiol Scand 2009, 53:828-829.

35. MCDonnell NJ: Cardiopulmonary arrest in pregnancy: two case reports of successful outcomes in association with perimortem Caesarean delivery. Br J Anaesth 2009, 103:406-409.

36. Bush MC, Nagy S, Berkowitz RL, Gaddipati S: Pregnancy in a persistent vegetative state: case report, comparison to brain death, and review of the literature. Obstet Gynecol Surv 2003, 58:738-748.

37. Farragher R, Marsh B, Laffey JG: Maternal brain death--an Irish perspective. Ir J Med Sci 2005, 174:55-59.

38. Mallampalli A, Guy E: Cardiac arrest in pregnancy and somatic support after brain death. Crit Care Med 2005, 33:S325-S331.

\section{Further reading}

1. ICNARC - Female admissions (aged 16-50 years) to adult, general critical care units in England, Wales and Northern Ireland, reported as 'currently 
pregnant' or'recently pregnant' 1 January 2007 to 31 December 2007 [http://www.oaa-anaes.ac.uk/assets/_managed/editor/File/Reports/ICNARC_ obs_report_Oct2009.pdf].

2. Martin SR, Foley MR: Intensive care in obstetrics: an evidence-based review. Am J Obstet Gynecol 2006, 195:673-689.

3. Williams J, Mozurkewich E, Chilimigras J, Van De Ven C: Critical care in obstetrics: pregnancy-specific conditions. Best Pract Res Clin Obstet Gynaecol 2008, 22:825-846.

4. Zeeman GG: Obstetric critical care: a blueprint for improved outcomes. Crit Care Med 2006, 34 (9 Suppl):S208-214.
5. Zeeman GG, Wendel GD Jr., Cunningham FG: A blueprint for obstetric critical care. Am J Obstet Gynecol 2003, 188:532-536.

doi:10.1186/cc10256

Cite this article as: Neligan PJ, Laffey JG: Clinical review: Special populations - critical illness and pregnancy. Critical Care 2011, 15:227. 